\title{
Particle tunneling through a polarizable insulator
}

\author{
Peter Nalbach ${ }^{1}$ and Walter Harrison ${ }^{2}$ \\ ${ }^{1}$ Department of Physics, ${ }^{2}$ Department of Applied Physics, \\ Stanford University, Stanford, California 94305, USA
}

(Dated: November 20, 2018)

\begin{abstract}
The tunneling probability between two leads connected by a molecule, a chain, a film, or a bulk polarizable insulator is investigated within a model of an electron tunneling from lead A to a state higher in energy, describing the barrier, and from there to lead B. To describe the possibility of energy exchange with excitations of the molecule or the insulator we couple the intermediate state to a single oscillator or to a spectrum of these, respectively. In the single-oscillator case we find for weak coupling that the tunneling is weakly suppressed by a Debye-Waller-type factor. For stronger coupling the oscillator gets stiff and we observe a suppression of tunneling since the effective barrier is increased. The probability for the electron to excite the oscillator increases with the coupling. In the case of a film, or a bulk barrier the behavior is qualitatively the same as in the single oscillator case. An insulating chain, as opposed to a film or a bulk connecting the two leads, shows an orthogonality catastrophe similar to that of an electronic transition in a Fermi gas.

PACS numbers: 73.63.-b, 73.23.Hk, 72.20.Dp
\end{abstract}

There is a vast interest in the electronic properties of molecular systems or tunnel junctions due to their potential use for electronic applications. Thereby, a tunnel junction might be a microscopic or macroscopic one-, two- or three dimensional insulator connecting two metal leads. As long as the molecule or the tunnel junction is not excited by the electron the conductance can be phenomenologically described by introducing a matrix element for tunneling through the barrier. However, the possibility to excite the barrier opens new paths for the electron, thus, enhancing the conductance. In recent experiment on molecular junctiond 1 the conductance shows several steps with increasing bias voltage between the two leads underlining the importance of vibrations excited by the tunneling electron 3 . We are solely interested in the influence of vibrations on the conductance and we do not restrict ourselves to weak coupling between the vibrations and the tunneling electron. In detail we investigate the probability for a tunneling electron to excite the molecule/tunnel junction. Therefore we introduce an intermediate state in the junction which itself is coupled to either a single oscillator to mimic the excitations of a molecule or to a spectrum of oscillators to mimic photonic excitations of a tunnel junction. A similar approach allowing two paths to a final state for a tunneling particle was used by Harrisont to investigate how the coupling destroys the coherence between two paths.

A Single Oscillator - The electron starts in lead A from an initial state $|0\rangle$ with energy $E_{0}$. This state is coupled to the intermediate electron state $|1\rangle$ with energy $E_{1} \geq E_{0}$ by the tunneling matrix element $V_{01}$. The intermediate state is also coupled by a matrix element $V_{1 \alpha} / \sqrt{M}$ to $M$ final states $|\alpha\rangle$ with $\alpha \geq 2$ in lead B. For sufficiently small tunneling matrix elements the transition probability $P_{0, \mathrm{f}}$ for the electron tunneling from $|0\rangle$ through $|1\rangle$ to a final state $|\alpha\rangle$ can be obtained by simple second-order time-dependent perturbation theory 5 as

$$
P_{0, \mathrm{f}}=\frac{2 \pi}{\hbar} \frac{1}{M} \sum_{\alpha}\left|\frac{V_{1 \alpha} V_{01}}{E_{1}-E_{0}}\right|^{2} \delta\left(E_{0}-E_{i}\right)=\frac{2 \pi V_{1 \alpha}^{2} V_{01}^{2}}{b \hbar \epsilon^{2}}
$$

with $\epsilon=E_{1}-E_{0} \gg V_{01}, V_{1 \alpha}$. For the summation over all final electron states we assumed a uniform density of states of the $M$ states within a band of bandwidth $b$ in lead B.

An interaction $H_{I}$ between the intermediate state and the excitations of a molecule can be mimicked by coupling the state $|1\rangle$ to a single harmonic oscillator: $H_{I}=$ $\gamma|1\rangle\langle 1| \hat{x}$ where $\gamma$ is a coupling constant and $\hat{x}$ is the displacement coordinate of the oscillator. The quantum mechanical oscillator states when the electron is in one of the leads are denoted as $\left|\phi_{i}\right\rangle$, where $i$ is the excitation number, whereas $\left|\psi_{i}\right\rangle$ is the oscillator state with $i$ excitations when the electron is in the intermediate state. The total state of the problem can be expressed by the states $\left|0, \phi_{i}\right\rangle,\left|1, \psi_{i}\right\rangle$ and $\left|2, \phi_{i}\right\rangle$ with energy $E_{\nu=0,1, \alpha}+i \hbar \omega$ (the zero-point energy is absorbed in the $E_{\nu}$ ).

We consider only initial states without excitations. The probability $P_{00, \mathrm{f} n}$ for tunneling from state $|0\rangle$ with zero excitations to a final state with $n$ excitations in the oscillator is modified from Eq. (11) as

$$
P_{00, \mathrm{f} n}=P_{0, \mathrm{f}} \cdot\left|\sum_{i} \frac{\epsilon}{\epsilon+i \hbar \omega}\left\langle\phi_{n} \mid \psi_{i}\right\rangle\left\langle\psi_{i} \mid \phi_{0}\right\rangle\right|^{2}
$$

where we sum over all possible numbers $i$ of virtual excitations in the oscillator in the intermediate state.

The oscillator states $\psi_{i}(x)=\phi_{i}\left(x-x_{0}\right)$ are shifted by

$$
x_{0}=\frac{\gamma}{m \omega^{2}}
$$

since an electron in the intermediate state shifts the potential minimum position of the oscillator. With the zero 
point fluctuation

$$
a_{0}=\sqrt{\frac{\hbar}{m \omega}}
$$

of the harmonic oscillator we obtain for the overlaps

$$
\begin{aligned}
& \left\langle\phi_{n} \mid \psi_{i}\right\rangle=\exp \left(-\frac{x_{0}^{2}}{4 a_{0}^{2}}\right) . \\
& \sum_{\substack{0 \\
0 \\
n-i \\
i<n}}^{n} \frac{(-1)^{j} \sqrt{\frac{i ! n !}{2^{i-n+2 j}}}}{j !(i-n+j) !(n-j) !}\left(\frac{x_{0}}{a_{0}}\right)^{i-n+2 j} .
\end{aligned}
$$

Assuming that the oscillator is in the ground state before and after tunneling the expressions simplify substantially and we obtain for the tunneling probability

$$
P_{00, \mathrm{f} 0}=P_{0, \mathrm{f}} \cdot\left|\sum_{i} \frac{1}{i !} W^{i} \frac{\epsilon}{\epsilon+i \hbar \omega}\right|^{2} \exp (-2 W),
$$

defining

$$
W=\frac{1}{2}\left(\frac{x_{0}}{a_{0}}\right)^{2} .
$$

The sum runs over the number $i$ of virtual excitations in the intermediate state. The contributions peak for $i \simeq W$ virtual excitations. In the weak-coupling limit, $W \ll 1$, tunneling without virtual excitations, $i=0$, dominates and the tunneling is influenced by a DebyeWaller type term $\sim \exp (-2 W)$. For strong coupling, $W \gg 1$, the contribution to the sum peaks at $i \sim W$ and noting $\sum_{i} W^{i} / i ! \sim e^{W}$ we obtain

$$
P_{00, \mathrm{fo}} \simeq P_{0, \mathrm{f}} \cdot\left|\frac{\epsilon}{\epsilon+W \hbar \omega}\right|^{2}=\frac{2 \pi}{\hbar b} \frac{V_{1 \alpha}^{2} V_{01}^{2}}{(\epsilon+W \hbar \omega)^{2}}
$$

which numerically turns out to be very precise when $W \gg 1$. The tunneling electron leaves the oscillator unmoved but its potential minimum position is shifted putting the oscillator in an excited state with an excitation energy $\frac{1}{2} m \omega^{2} x_{0}^{2}=W \hbar \omega$. Accordingly, the barrier is effectively raised by this energy.

Naturally, we would assume that the crossover at $W \sim 1$ coincides with the region where $P_{00, \text { fo }}$ falls from its value without coupling to an oscillator to a strongly suppressed probability. However, Eq. (8) shows that even for $W \gg 1$ the coupling to the oscillator only influences the tunneling probability substantially for $W \hbar \omega>\epsilon$. Our perturbative approach limits us to energies smaller than the barrier heights. Therefore we cannot investigate the strong coupling limit $W \hbar \omega \geq \epsilon$ and we are confined to oscillator frequencies $\omega<\epsilon / \hbar$.

Defining a number $n_{r}$ with

$$
n_{r} \hbar \omega=\epsilon
$$

we find that $P_{00, \text { fo }}$ is a function of $W / n_{r}$ instead of $W$ and we are restricted to the effective weak coupling limit $W / n_{r} \leq 1$.
If we interpret the virtual excitation energy $W \hbar \omega$ as the energy put into the oscillator during tunneling we expect that in the resonant case when $W=1$ the probability for exciting the oscillator will be maximal. However, we saw that for $\hbar \omega \leq \epsilon$ the influence on the electron from the coupling to the oscillator increases with $W / n_{r}$. We numerically find that the probability $P_{00,21}$ for exciting the oscillator with a single excitation increases linear with $W / n_{r}$ (quadratic with the coupling $\gamma$ between electron and oscillator) and the probability $P_{00,2 n}$ decreases for a higher number of excitations $n$. If we expand $\epsilon /(\epsilon+i \hbar \omega) \approx 1-i \hbar \omega / \epsilon$ in Eq. (2) up to first order in $\hbar \omega / \epsilon$ we obtain

$$
P_{00, \mathrm{f} n} \simeq P_{0, \mathrm{f}} \cdot\left|\delta_{n 0}+\left\langle\phi_{n}\left|\frac{\gamma \hat{x}}{\epsilon}\right| \phi_{0}\right\rangle\right|^{2}
$$

which shows the quadratic behavior with the coupling $\gamma$. Summing over all possible numbers $n$ of excitations we get for the probability to excite the oscillator $P_{00, \mathrm{f} n \geq 1} \approx$ $\left(\gamma a_{0} / \epsilon\right)^{2} P_{0, \mathrm{f}}$. This approximation has the same limit as discussed before namely that the virtual energy $W \hbar \omega$ of the mainly contributing tunneling path is smaller than the barrier heights $\epsilon \geq W \hbar \omega$. Therefore this result holds only for couplings where $\left(\gamma a_{0} / \epsilon\right)^{2} \leq(2 \hbar \omega / \epsilon) P_{0, \mathrm{f}}$.

Multiple Oscillators - In the next step we introduce a spectrum of oscillators coupled to the tunneling electron to investigate the tunneling of an electron through a tunnel junction, for example a linear chain of atoms as illustrated in Fig. 11. Assuming that all bonding states between the atoms of the chain are occupied by electrons, the tunneling electron hops to an anti-bonding state between two atoms. The electron repels with force $F$ the nearest neighbors resulting in a coupling

$$
H_{I}=-|1\rangle\langle 1| \cdot F \cdot\left(\delta x_{m+1}-\delta x_{m}\right)
$$

of an electron in state $|1\rangle$ to the displacement $\delta x_{m+1}$ and $\delta x_{m}$ of the nearest neighbors. For a chain of $N$ atoms and lattice constant $d$ we obtain, using fixed boundary conditions at position $r=0$ and $r=(N+1) d, N$ phonon modes with $\delta x_{m}=\sqrt{2 / N} \sum_{k} u_{k} \sin \left(k x_{m}\right)$ and

$$
k=k_{n}=\frac{n}{N+1} \frac{\pi}{d} \quad \text { with } \quad n=1 . . N .
$$

It is convenient to take $N$ even and the anti-bond $m=$ $N / 2$. Then the coupling between the electron and the phonons turns out to be

$$
H_{I}=|1\rangle\langle 1| \cdot \sum_{k_{n}} \lambda_{k_{n}} u_{k_{n}}
$$

with the coupling constants

$$
\lambda_{k_{n}}=\left\{\begin{array}{ll}
2 F \cdot \sqrt{\frac{2}{N}} \sin \left(\frac{k_{n} d}{2}\right) & : n \text { odd } \\
0 & : n \text { even }
\end{array} .\right.
$$

In the following we use the indices $k_{n}, k$ and $n$ interchangeably. The coupling constant $\lambda_{n}$ between the electron and the mode with wave vector $k_{n}$ is proportional 

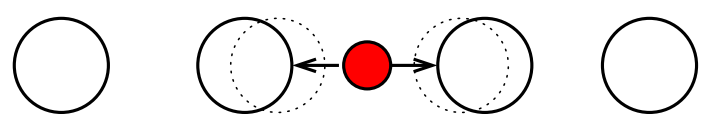

FIG. 1: A linear chain of atoms is illustrated in which a tunneling electron might hop to an anti-bond between atom $m$ and $m+1$ repelling both neighbors.

to $1 / \sqrt{N}$, so in the thermodynamic limit $(N \longrightarrow \infty)$ the coupling effects estimated by $\sum_{n} \lambda_{n}^{2}$ stay finite.

In the case of a single oscillator the behavior of the system was determined by $W=\frac{1}{2}\left(x_{0} / a_{0}\right)^{2}$. Since $\lambda_{n} \sim$ $1 / \sqrt{N}$ in the case of many phonons each single mode is weakly coupled to the electron for large $N$. However, the influence to the tunneling electron is now determined by the sum over all modes $W=\frac{1}{2} \sum_{n}\left(x_{n} / a_{n}\right)^{2}$ with $x_{n}=\lambda_{k_{n}} /\left(m \omega_{n}^{2}\right)$ and $a_{n}=\sqrt{\hbar /\left(m \omega_{n}\right)}$. The equations given above are for a one-dimensional chain but they are easy to generalize to two and three dimensions. It is a little intricate to work out the coupling but essentially the coupling constants $\lambda_{k}$ look the same and only the density of phonon modes $g(k)$ depends on the dimensionality, $g(k) \propto k^{d-1}$. This turns out to give a striking difference between the one dimensional and the other cases.

With no phonon in the initial state $\left\{m_{k}=0\right\}$ we find for the transition probability to the final state with $l_{k}$ excitations in mode $k_{n}$

$$
\begin{gathered}
P_{0\left\{m_{k}=0\right\}, \mathrm{f}\left\{l_{k}\right\}}=P_{0, \mathrm{f}} . \\
\left|\sum_{\left\{i_{k}\right\}} \frac{\epsilon}{\epsilon+\sum_{k} i_{k} \hbar \omega_{k}} \prod_{k}\left\langle\phi_{l_{k}}(k) \mid \psi_{i_{k}}(k)\right\rangle\left\langle\psi_{i_{k}}(k) \mid \phi_{0}(k)\right\rangle\right|^{2}
\end{gathered}
$$

where $\phi_{l_{k}}(k)$ and $\psi_{l_{k}}(k)$ are the wavefunctions of the unshifted and shifted mode $k$ with $l$ excitations respectively. The sum $\sum_{\left\{i_{k}\right\}}$ sums over all possible total transient states with virtual excitation numbers $i_{k}$ for each mode. For each of these states the product $\prod_{k}$ over the various overlaps calculates the total overlap between the initial and the transient state times the overlap of the transient and the final state.

Let us focus first on the probability $P_{0\left\{m_{k}=0\right\}, f\left\{l_{k}=0\right\}}$ for tunneling without final state excitations. The contribution $A_{0}$ from the tunneling 'path' without virtually exciting any phonon is given by the overlap between the ground states of the unshifted and shifted oscillator modes

$$
A_{0}=\prod_{k}\left\langle\phi_{0}(k) \mid \psi_{0}(k)\right\rangle\left\langle\psi_{0}(k) \mid \phi_{0}(k)\right\rangle=e^{-\frac{1}{2} \sum_{n} \frac{x_{n}^{2}}{a_{n}^{2}}} .
$$

Einstein Approximation - An Einstein approximation leaves us essentially with $N$ modes with the same frequency $\omega_{E}$ and coupling constant $\gamma$. We find

$$
W=\frac{1}{2} \sum_{n} \frac{x_{n}^{2}}{a_{n}^{2}}=\frac{1}{2} \frac{\gamma^{2}}{\hbar m \omega_{E}^{3}} .
$$

which is identical to the single oscillator result. For the contributions $A_{m}$ from tunneling paths involving $m$ virtual excitations we obtain

$$
A_{m}=W^{m} \frac{1}{m !} e^{-W} \cdot \frac{\epsilon}{\epsilon+m \hbar \omega_{D}} .
$$

The factorial $1 / m$ ! results from the fact that, once we chose $m$ oscillators to put the $m$ virtual excitation in, there are still $m$ ! ways of distributing the virtual excitation to the oscillators. Physically, however all these $m$ ! ways are identical. Technically it is convenient to sum over all these and we obtain $m ! A_{m}$ which we then divide by the factorial to get Eq. (15). Note also that $A_{m}$ includes only tunneling path where all $m$ excitations are in $m$ different modes. Tunneling paths with more than one excitation in a mode are suppressed for large $N$ by factors $1 / N$. The probability $P_{0\left\{m_{k}=0\right\}, f\left\{l_{k}=0\right\}}$ for tunneling without final state excitations thus is equivalent to Eq. (6). An Einstein spectrum yields the same tunneling probability as a single oscillator if no mode is excited.

The probability for tunneling through the chain and exciting mode $j$ with a single excitation is given by

$$
P_{00, \mathrm{f}_{j}}=\left(\frac{x_{j}}{a_{j}}\right)^{2} e^{-\frac{x_{j}^{2}}{2 a_{j}^{2}}} P_{00,20}^{\prime} .
$$

$P_{00,20}^{\prime}$ is the probability for the electron tunneling through the chain without final state excitations calculated without including mode $j$. Since a single mode does not change results appreciably we take $P_{00,20}^{\prime} \simeq P_{00,20}$. We sum $j$ over all modes. The exponential is unity for large $N$ and we use Eq. (14) to find for the probability to excite $n$ modes

$$
P_{00, \mathrm{f} n}=\frac{1}{n !} W^{n} P_{00,20} .
$$

Note that contributions from tunneling path with more than one excitation in a single mode are suppressed. The factorial $n$ ! has the same origin as $m$ ! in Eq. (15). For $W<1$ tunneling through the chain without exciting it and without using virtual excitations dominates. However, for $W>1$ the tunneling electron uses predominantly $W$ virtual excitations to tunnel and the chain has most likely $W$ excitations once the electron tunneled through. This shows a difference from the single oscillator case where in the strongly coupled regime, $W>1$, the oscillator was not excited by the tunneling electron. Only for $W / n_{r}$ near one excitations are likely. To understand this we note that each single mode in the many oscillator case is weakly coupled. The probability to excite some is only finite because there are many modes.

Debye Approximation - Within a Debye approximation we assume a spectrum of modes with linear dispersion $\omega=v k$ with the speed of sound $v$. The Debye cut-off $k_{D}=(N / N+1)(\pi / d)$ is chosen to give the correct number of modes. It leads qualitatively to the same results as an Einstein approximation for a two and a three dimensional tunnel junction since their phonon spectra favor 
high frequency modes and $\omega_{E}$ is replaced by an average frequency. We therefore focus on tunneling through a chain of atoms. We further restrict the following considerations to the probability for tunneling without final state excitations since the probability for tunneling with final state excitations follows then from Eq. (17).

The chain has a constant density of states and we find

$$
W=\frac{1}{2} \sum_{n} \frac{x_{n}^{2}}{a_{n}^{2}}=\frac{k_{c}}{k_{D}} \sum_{j=1}^{N / 2} \frac{1}{j} \sim \frac{k_{c}}{k_{D}} \ln (N)
$$

with $k_{c}=(F d)^{2} /\left(\hbar m v^{3}\right)$. For large $N$ tunneling through the chain without the help of virtual excitations is suppressed by $\exp (-2 W) \sim \exp \left(-2\left(k_{c} / k_{D}\right) \ln (N)\right)=$ $N^{-\left(2 k_{c} / k_{D}\right)}$. In the thermodynamic limit, $N \rightarrow \infty$, this approaches zero. This behavior is similar to the 'orthogonality catastrophe' described by Anderson 6 for an electron tunneling into a Fermi gas which has a constant density of states near the Fermi surface.

We now investigate the contribution $A_{m}$ from a tunneling path involving $m$ virtual excitations. The key question is whether there is a dominant tunneling path (a $m$ with $A_{m}$ maximal) and what would be its effective virtual energy $\sum_{k} i_{k} \hbar \omega_{k}$ (compare Eq. (13)). We suggest that the contribution of mode $j$ to the effective virtual energy is determined by a factor $\left(x_{j}^{2} / a_{j}^{2}\right)$ since the same factor determined in the case of tunneling with one final state excitation the contribution of the tunneling path with mode $j$ finally excited (compare Eq. (16)). Since $A_{m}$ involves $m$ virtual excitations we introduce further a 'normalizing' factor $m / W$ and find

$$
A_{m}=e^{-W} W^{m} \frac{1}{m !} \frac{\epsilon}{\epsilon+\frac{1}{2} \sum_{j} \frac{m x_{j}^{2}}{W a_{j}^{2}} \hbar \omega_{j}} .
$$

For the energy of the $m$ virtual excitations we find

$$
\sum_{j} \frac{m x_{j}^{2}}{W a_{j}^{2}} \hbar \omega_{j}=\frac{m}{W} \hbar \omega_{c} .
$$

Numerically we find that this approximation holds when the energy of the $m$ virtual excitations does not exceed the barrier heights. For large finite $N$ we find that $W$ tends logarithmically to infinity. However, for $W \gg 1$ the probability for tunneling without final state excitations is simply given by the tunneling probability through an effective barrier (compare Eq. (8)) enhanced by the energy of the virtual excitations of the dominating tunneling path. The dominating path involves $W$ virtual excitations leading to an energy $\hbar \omega_{c}$ of the virtual excitations which is independent of $W$. Thus the probability for tunneling through the chain without final state excitations is finite and independent of the length $N$.

Note that the maximal virtual energy $W \hbar \omega_{D}$ possibly used in a tunneling event with $W$ virtual excitations increases logarithmically with the number $N$ of modes. Once this energy $W \hbar \omega_{D}$ exceeds the barrier heights $\epsilon$ the numerical investigation showed that our approximation, Eq. (19), fails. However at the same point our approach using second order perturbation theory is questionable. We therefore believe that our approximative result holds in the thermodynamic limit and that tunneling through a chain without final state excitations is possible.

In conclusion, we first investigated tunneling through a barrier with a coupling to a single oscillator. The result can be characterized by a coupling strength $W$ given by the square of the ratio of the classical shift of the oscillator to its zero-point fluctuation. For weak coupling, $W<1$, tunneling without final state excitations is suppressed by a factor $e^{-2 W} \approx 1-2 W$. For strong coupling, $W>1$, tunneling is suppressed by an increase in the tunneling barrier height by an energy $W$ times the vibrational energy $\hbar \omega$. The probability for tunneling with final state excitations increases in proportion to $W$. We then introduced a barrier with coupling to a spectrum of $N$ modes, with couplings proportional to $1 / \sqrt{N}$. It is shown that in an Einstein approximation, where all modes have the same frequency $\omega_{E}$, the results are essentially the same. The same holds true for two or three dimensional Debye spectra where the frequency is proportional to the wave number. However, a one-dimensional chain shows an orthogonality catastrophe similar to that of an electronic transition in a Fermi gas since $W \propto \ln (N)$. The probability for tunneling without final state excitations and without the help of virtual excitations is suppressed by a factor $1 / N$ raised to a finite power. However, our investigation suggests that the probability for tunneling without final state excitations but with the help of virtual excitations stays finite for $N \rightarrow \infty$.

P. Nalbach was sponsored by the Alexander von Humboldt foundation and the U.S. Dept. of Energy grant DE-FG03-90ER45435-M012.
1 N.B. Zhitenev, H. Meng, and Z. Bao, Phys, Rev. Lett. 88, 226801 (2002)

${ }^{2}$ H. Park, J. Park, A.K.L. Lim, E.H. Anderson, A.P. Alivisatos and P.L. McEuen, Nature 407, 57 (2000)

3 D. Boese and H. Schoeller, Europhys. Lett. 54, 668 (2001)

${ }^{4}$ W.A. Harrison, Phys. Rev. B 50, 8861 (1994)
${ }^{5}$ For example, L.D. Landau and E.M. Lifshitz, in Quantum Mechanics, Non-relativistic Theory, translated by J.B. Sykes and J.S. Bell (Pergamon, London, 1958), p. 150.

${ }^{6}$ P.W. Anderson, Phys. Rev. Lett. 18, 1049 (1967) 